\title{
Microcirculation in healing and healthy Achilles tendon assessed with invasive laser doppler flowmetry
}

\author{
Erica Domeij Arverud ${ }^{1}$ \\ Olof Persson-Lindell ${ }^{2}$ \\ Fredrik Sundquist ${ }^{2}$ \\ Fausto Labruto ${ }^{3}$ \\ Gunnar Edman 4 \\ Paul W. Ackermann²
}

1 Department of Orthopedic Surgery, Danderyd Hospital, Stockholm, Sweden

2 Department of Orthopedic Surgery, Karolinska University Hospital, Stockholm, Sweden

3 Section of Diagnostic Imaging, Department of Molecular Medicine and Surgery, Karolinska Institutet, Stockholm, Sweden

4 Department of Neurobiology, Care Sciences, and Society, Centre of Family Medicine (CeFAM), Karolinska Institutet, Stockholm, Sweden

Corresponding author:

Paul W. Ackermann

Department of Orthopedics, Karolinska University Hospital

A2:00, 17176 Stockholm, Sweden

E-mail: paul.ackermann@karolinska.se

\section{Summary}

Introduction: Achilles tendon (AT) rupture exhibits a prolonged healing process with varying clinical outcome. Reduced blood flow to the AT has been considered an underlying factor to AT rupture (ATR) and impaired healing. In vivo measurements using laser Doppler flowmetry (LDF) may be a viable method to assess blood flow in healthy and healing AT.

Methods: 29 persons were included in the study; 9 being ATR patients and 20 healthy subjects without any prior symptoms from the AT. Invasive LDF was used to determine the post-occlusive reactive hyperemia (PORH) in the paratenon after 15 minutes of occlusion of the lower extremities. ATR patients were examined two weeks post-operatively.

Results: LDF-assessments demonstrated a significantly different $(p<0.001)$ PORH response in the healing- versus intact- and control AT. In the healing AT, a slow, flattened PORH was observed compared to a fast, high peak PORH in intact, healthy AT.
Conclusion: in vivo LDF appears to be a feasible method to assess alterations in blood flow in healing and intact AT. The healing ATs capability to react to an ischemic period is clearly impaired, which may be due to the trauma at injury and/or surgery or degenerative changes in the tendon.

KEY WORDS: blood flow, circulation, hyperemia, regeneration, rupture, tendon.

\section{Introduction}

In patients with acute Achilles tendon (AT) rupture the time until healing is protracted, associated with a high degree of complications and variable outcome ${ }^{1-3}$. This may be due partly to a sparse blood circulation during AT healing of the immobilized limb4.

The blood supply of the AT originates both from the intrinsic proximal muscle-tendon junction and distal bone insertion and from the extrinsic system, which delivers blood along the length of the tendon through the paratenon ${ }^{5}$. Several studies have shown the extrinsic system to play a more prominent role in the blood supply of the human $A^{5-8}$. Still, however, the in vivo AT extrinsic microcirculation remains largely unknown both in healthy and healing AT patients.

Microcirculation can be assessed using established methodology, e.g. Laser Doppler flowmetry (LDF) ${ }^{9} 10$. LDF calculates blood flow by measuring the shift in light frequency, i.e. Doppler shift, which occurs when a transmitted laser beam hits moving red blood cells ${ }^{11}$. Reproducible LDF-data can be established by quantifying rapid changes in perfusion, during certain standardized events ${ }^{9}, 11,12$, for example post occlusive reactive hyperemia $(\mathrm{PORH})$. This method is widely used and suitable for determining deficiencies in limb microcirculation ${ }^{12}$. To our knowledge, there have been no studies using invasive LDF combined with PORH-methodology in order to establish the extrinsic blood flow in the paratenon in neither the healthy nor the healing AT.

An earlier study on experimental Achilles tendon rupture indicated that the blood flow to the tendon was not still restored at two weeks after injury ${ }^{13}$. In this study we hypothesized that the microcirculatory blood flow in the Achilles paratenon could be accurately assessed using invasive LDF and that the blood flow would be altered during early human Achilles tendon repair as compared to a healthy Achilles tendon. Moreover we hypothesized that the contralateral 
Achilles tendon to the ruptured may exhibit an altered microcirculatory blood flow as compared to a healthy Achilles tendon.

To answer these questions microcirculatory blood flow in the Achilles paratenon was assessed with invasive LDF combined with $\mathrm{PORH}$-methodology bilaterally on patients at 2 weeks post-operative Achilles tendon rupture (ATR) and on healthy controls.

\section{Material and methods}

\section{Patients}

Forty-one patients were eligible for inclusion and twenty-nine of these patients ( 9 with ATR and 20 healthy volunteers) were included in this experimental study (Fig. 1).

Patients between 18-75 years with acute unilateral ATR, operated on within 72 hours were recruited from a larger study at the Hospital (Clinicaltrials.gov ID NCT01317160) that examined the prevalence and prevention of DVT after ATR, which has been published $^{14}$. Recruitment was executed between January and June 2012. The following exclusion criteria were applied: inability to give consent for participation, ongoing treatment with anticoagulants, known allergy to contrast media, planned follow-up at another hospital, inability to follow instructions, known renal insufficiency, heart failure with pitting edema, thrombophlebitis, thromboembolic disease during the last 3 months, surgery during the last month, known malignancy, hemophiliacs, pregnancy, treatment with high dosage of aspirin or unwillingness to participate in the study. Twenty healthy subjects, with no history of AT pathology were recruited. The same exclusion criteria were applied for the healthy volunteers as for the patients. Totally 41 persons were included in the study. Of these, 20 were healthy subjects and 21 were patients with ATR. Twelve of the patients were excluded from the LDF-study, due to declining participation $(n=4)$ or DVT $(n=8)$ (Fig. 1). Three of healthy subjects were excluded due to motion artefacts during the examination. All eligible subjects received standardized verbal and written information about the trial, and gave written informed consent to participate in the study. The present study was conducted with approval by the Regional Ethical Review Committee and conforms with the ethical standards of the journal' ${ }^{15}$.

The patients with ATR, age 36.6 (25-44), were significantly older than the healthy volunteers, age 27.1 (22-43). There was also a near significant difference in gender between the two groups $(p=0.066)(T a b .1)$.

\section{Surgical procedure and post-operative treatment protocol}

The surgical procedure was performed under local anaesthesia (Marcain $5 \mathrm{mg} / \mathrm{ml}$ with adrenaline), without the use of a tourniquet. A longitudinal $5-10 \mathrm{~cm}$ medial skin incision was made and the paratenon was incised on the midline. The tendon stumps were end-to-end sutured using modified Kessler suture technique with two 1-0 polydioxanone (PDS II) sutures. Thereafter, the paratenon and fascia cruris were separately sutured using 3-0 vicryl, and the skin was closed with 3-0 ethilon. All sutures were supplied by Ethicon, Somerville, NJ, USA. After completion of surgery, patients were randomly assigned by computer-generated random numbers in permuted blocks of 4 to undergo either standard plaster cast treatment alone or plaster cast treatment with adjuvant calf intermittent pneumatic compression (IPC) treatment beneath an orthotic device. Patients in the IPC group $(n=5)$ received two weeks of 6 hours daily bilateral calf IPC (Venaflow Elite, DJO, Vista, California, USA). As the IPC-device cycles, the distal chamber inflates to $73 \mathrm{~mm} \mathrm{Hg}$ over a 0.5 -second period. During the last 0.2 second of this period, the proximal chamber inflates to $63 \mathrm{~mm} \mathrm{Hg}$ and then settles at 45 $\mathrm{mm} \mathrm{Hg}$. After six seconds of inflation the cuff deflates, and the cycle is repeated every minute. The patients were instructed to apply the intermittent

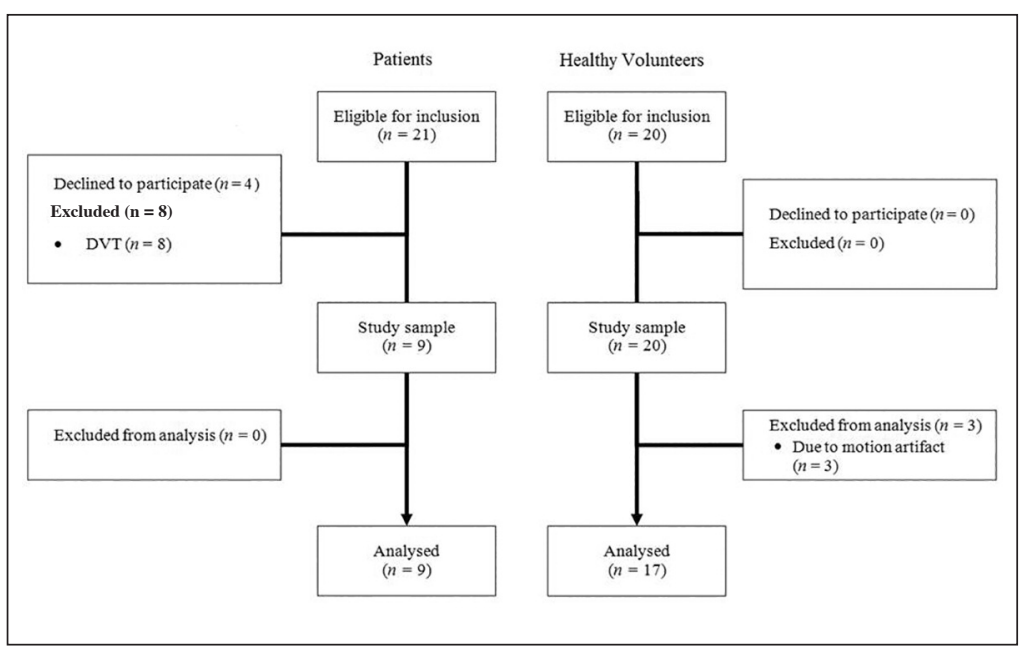

Figure 1. Enrolment. Overview of the study group of patients with Achilles tendon rupture and healthy volunteers. Abbreviations: DVT = deep vein thrombosis. 
Table 1. Characteristics of the study population.

\begin{tabular}{llll}
\hline Variable & Patients $(\mathbf{n}=\mathbf{9})$ & Healthy subjects $(\mathbf{n}=\mathbf{2 0})$ & -value \\
\hline Mean age $(\mathrm{yrs})$ (range) & $36.6(25-44)$ & $27.1(22-43)$ & $0.001^{*}$ \\
Male $(\mathrm{n}, \%)$ & $9(100)$ & $13(65)$ & $0.066 \dagger$ \\
\hline${ }^{*}:$ Independent samples test & & & \\
$\dagger$ : Fishers exact test & & &
\end{tabular}

pneumatic compression therapy during the time they were sedentary, i.e. sitting or lying in bed sleeping, 6 hours at a minimum daily. Patient compliance was registered by the patient and by the device. Intermittent pneumatic compression treatment was discontinued two weeks post-operatively.

The control group $(n=4)$ received a below-knee plaster cast with the ankle in 30 degrees equinus position in the outpatient clinic shortly after the completion of surgery, and were instructed to be non-weight bearing with crutches during the first two weeks. This is the current standard procedure used in our department and this was chosen to standardize the plaster cast application.

All patients were prescribed paracetamol 500 mg/ codeine $30 \mathrm{mg}$ to cope with the post-operative pain. No pharmacological anti-inflammatory or thromboprophylactic drugs were given to the patients post-operatively.

\section{Laser Doppler Flow (LDF)}

Two weeks post-surgery the blood flow in the tendon was evaluated with LDF. Before the LDF examination all patients were screened for DVT in the operated leg by unilateral color duplex sonography (CDS) using a Philips CX 50 (Philips Medical Systems, Andover, MA, USA). Patients diagnosed with deep vein thrombosis were excluded from the LDF study. A laser Doppler perfusion monitor (PF5010, LDPM Unit, Perimed $A B$, Järfälla, Sweden) with an invasive needle probe (Probe 402, Perimed AB, Järfälla, Sweden) was used to record changes in blood flow. The data obtained was presented as perfusion units (PU). The instrument was calibrated using the manufacturer's aqueous suspension.

All patients and healthy subjects were examined under the same circumstances, i.e. same surrounding temperature and minimized social interaction. The patients and subjects were placed on a gurney in prone position with their legs fully relaxed. Tourniquets were placed on each calf immediately below the knee. Local anaesthetic without adrenaline (Xylocain $^{\circledR}$, Astra Zeneca, $10 \mathrm{mg} / \mathrm{ml}$ ) was injected on the lateral side of each heel. Under ultrasound guidance (CX50 Ultrasound system, Philips, Netherlands), a radiologist introduced a 22-gauge catheter, $2 \mathrm{~cm}$ proximal to the calcaneus bone, into the peritendinous space 1-2 $\mathrm{mm}$ ventral to the Achilles tendon. The blood flow in the paratenon has been shown to reflect the supply to the tendons and also the intratendinous blood flow, therefore measurements were performed with the probes placed in the paratenon ${ }^{16}$. Thereafter the laser Doppler probes were inserted into the lumen of the catheters and the catheters were fixed using Tegaderm (3M, Minnesota, USA). After these procedures a baseline registration of the blood flow was performed during at least 10 minutes. After the baseline registration the tourniquet was simultaneously inflated to $300 \mathrm{~mm} \mathrm{Hg}$ to obtain supra-systolic pressure, thereby inducing a vascular occlusion. Vascular occlusion was kept for 15 minutes, thereafter the postocclusive reactive hyperemia $(\mathrm{PORH})$ was recorded during 15 minutes ${ }^{12}$.

The data was automatically stored in a Windowsbased software $\left(\right.$ PeriSoft ${ }^{\odot}$ Version 2.5.5, Perimed, Järfälla, Sweden). The data was stored as 32 data points per second and mean value of every second was exported to Microsoft Excel ${ }^{\odot}$ (Version 2007). The resting flux (RF) and biological zero (BZ) were calculated by extracting the median value during the longest artifact free and representative part of the baseline and occlusion ${ }^{17}$. To calculate the maximum flux (MF), the time to resting flux $\left(t_{R F}\right)$, the time to maximum flux ( $t_{M F}$ ) and the time to half recovery $\left(t_{H R}\right)$, during $\mathrm{PORH}$, the data was smoothed with a 21s- moving average. The protocol for the data management was based on previous findings by Morales et al. (2005). All parameters are presented in Table 2. The time variables $t_{\mathrm{RF}}, \mathrm{t}_{\mathrm{MF}}, \mathrm{t}_{\mathrm{HR}}$ as well as the ratio between MF and RF have all demonstrated significant differences between patients with peripheral arterial obstructive disease and healthy controls ${ }^{12}$. Thus, peripheral arterial obstructive disease demonstrate longer time variables and lower ratio of MF/RF.

Since the PORH in the healing AT from patients with post-operative adjuvant intermittent pneumatic compression and standard plaster cast treatment did not exhibit any significant differences $(p>0.05)$ the data from these patients have been pooled and the results will be presented as PORH in the ruptured, healing AT.

\section{Statistics}

All data were presented as mean values, standard error mean (SEM) and standard deviation. Repeated measures analysis of variance was used for analysis of all repeated measurements. Differences between the groups were analysed with the nonparametric Mann-Whitney $U$ test, and differences between the injured and intact legs with Wilcox on signed ranks test. These tests were used since the data was considered skewed. A value of $p<0.05$ (two-tailed) was 
Table 2. Definitions of variables used to study microcirculation.

\begin{tabular}{ll}
\hline Variable & Explanation \\
\hline $\mathbf{R F}(P U)$ & Resting flux: the median flux value obtained during baseline \\
$\mathbf{B Z}(\mathbf{P U})$ & Biological zero: the median flux value obtained during occlusion \\
$\mathbf{M F}(\mathrm{PU})$ & Maximum flux: the highest flux value obtained during PORH \\
$\mathbf{t}_{0}$ & Start of PORH \\
$\mathbf{t}_{\mathrm{RF}}(\mathbf{s})^{*}$ & Time to resting flux. the time from $\mathrm{t}_{0}$ until $\mathrm{RF}$ is reached \\
$\mathbf{t}_{\mathrm{MF}}(\mathbf{s})^{*}$ & Time to maximum flux: the time from $\mathrm{t}_{0}$ until $\mathrm{MF}$ is reached \\
$\mathbf{t}_{\mathrm{HR}}(\mathbf{s})^{*}$ & Time to half recovery: the time from $\mathrm{t}_{0}$ after $\mathrm{t}_{\mathrm{MF}}$ until $\frac{\mathrm{MF}+\mathrm{RF} \text { is reached }}{2}$ \\
$\mathbf{M F / R F ^ { * }}$ & Ratio of maximum flux and resting flux \\
\hline
\end{tabular}

Abbreviations: $\mathrm{PU}=$ Perfusion Unit; $\mathrm{PORH}=$ Post-occlusive reactive hyperemia.

* These variables have demonstrated significant differences in patients with peripheral arterial obstructive disease.

considered statistically significant. SPSS program (SPSS, Inc., Chicago, III.) was used for statistical analysis.

\section{Results}

\section{PORH in intact, external control AT}

The typical post-occlusive reactive hyperaemic $(\mathrm{PORH})$ response in the intact, healthy external control AT started with a fast rise in flux value followed by a quick recovery to baseline (Fig. 2). This response to occlusion was observed in all included intact, healthy ATs.

\section{PORH in ruptured, healing AT}

The nature of the PORH response in the healing AT, at two weeks post rupture, was significantly different from that of the intact, healthy AT. In contrast to the fast, high peak flux value after the occlusion observed in the healthy ATs (Fig. 2), a slow, flattened $\mathrm{PORH}$ was observed in the healing AT (Fig. 3). This difference in $\mathrm{PORH}$ was confirmed by the statistical significant difference seen in the parameters time to resting flux ( $\left.\mathrm{t}_{\mathrm{RF}}\right)(p<0.001)$ and maximum flux (MF)/resting flux (RF) $(p<0.001)$ (Tab. 3).

\section{PORH in intact, internal control AT}

The PORH response in the healing AT was also significantly different from the contralateral internal control AT. Thus, time to resting flux $\left(t_{R F}\right)$ was in the healing AT $131.4 \mathrm{~s}$, compared to $17.8 \mathrm{~s}$ in the internal control $(p<0.05)$. The ratio MF/RF was higher in the internal controls compared to the healing ATs $(p<$ 0.01) (Tab. 3).

Interestingly, the PORH response in the AT of the intact, internal control leg also demonstrated differences compared to the intact, external control. Hence, the biological zero was lower in the internal control AT compared to those of the external controls $(p<0.05)$. The ratio MF/RF was higher in the internal control- as compared to the external control ATs $(p<0.05)$ (Tab. 3). Moreover, there were more significant differences in $\mathrm{PORH}$ response between the healing AT and the external controls as compared to differences seen between the healing AT and internal controls.

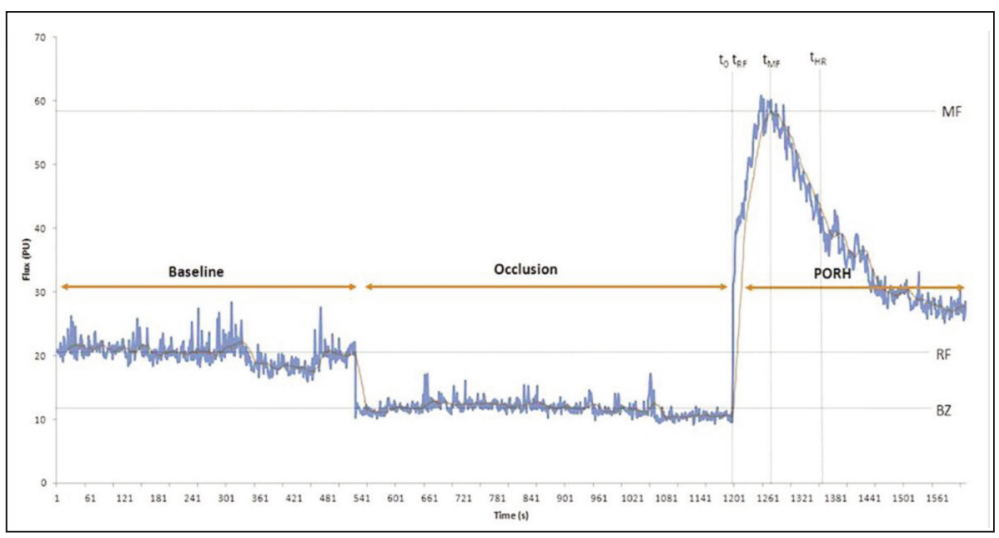

Figure 2. LDF-measurement on a healthy AT. The blue line is the signal averaged over 1 second. The brown line is the 21 second moving median. Abbreviations: $\mathrm{BZ}=$ Biological zero, $\mathrm{LDF}=$ Laser Doppler flowmetry, MF = Maximum flux, $\mathrm{PU}=$ Perfusion units, RF = Resting flux, $t_{0}=$ Start of post occlusive reactive hyperemia, $t_{\mathrm{RF}}=$ Time to resting flux, $t_{\mathrm{MF}}=$ Time to maximum flux, $\mathrm{t}_{\mathrm{HR}}=$ Time to half recovery. 


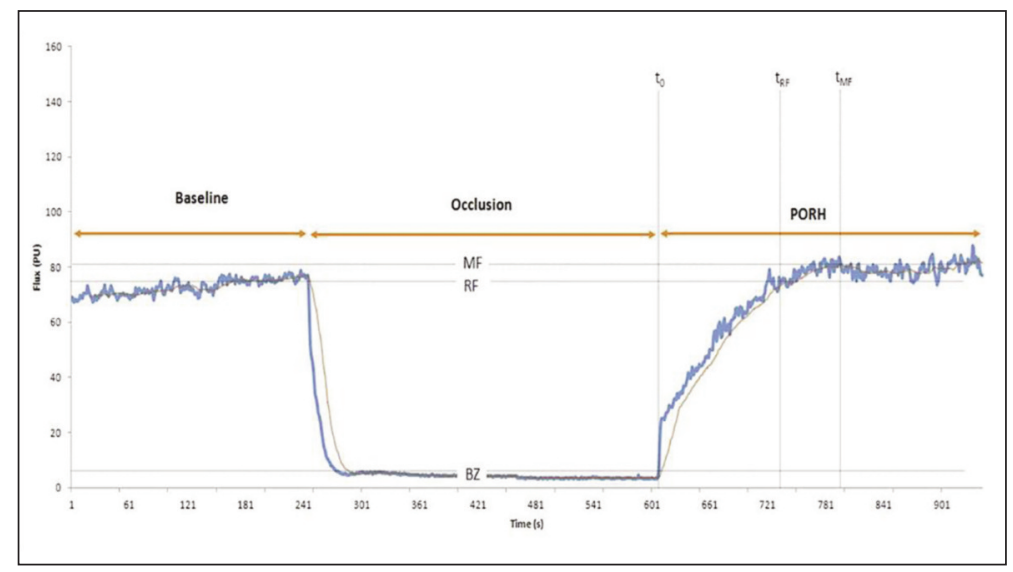

Figure 3. LDF-measurement on a healing AT. The blue line is the signal averaged over 1 second. The brown line is the 21 second moving median. Abbreviations: AT $=$ Achilles tendon, $B Z=$ Biological zero, LDF = Laser doppler flowmetry, MF = Maximum flux, $\mathrm{PU}=$ Perfusion units, RF $=$ Resting flux, $t_{0}=$ Start of post occlusive reactive hyperemia, $t_{\mathrm{RF}}=$ Time to resting flux, $\mathrm{t}_{\mathrm{MF}}=$ Time to maximum flux .

Table 3. Laser Doppler Flowmetry data in acute ATR patients and healthy subjects.

\begin{tabular}{|c|c|c|c|c|c|c|c|c|c|}
\hline \multirow[b]{2}{*}{ Parameters } & \multirow[b]{2}{*}{ Ruptured } & \multicolumn{2}{|c|}{$\begin{array}{l}\text { ATR patients } \\
\qquad(n=9)\end{array}$} & & \multirow{2}{*}{$\begin{array}{c}\text { Ruptured } \\
\text { versus } \\
\text { Intact, } \\
\text { internal } \\
\text { control } \\
\text { V P }\end{array}$} & \multicolumn{2}{|c|}{$\begin{array}{l}\text { Healthy subjects } \\
\qquad(n=20)\end{array}$} & \multirow{2}{*}{$\begin{array}{l}\text { Ruptured } \\
\text { versus } \\
\text { Intact, } \\
\text { external } \\
\text { control } \\
\text { P }\end{array}$} & \multirow{2}{*}{$\begin{array}{c}\text { Intact, } \\
\text { internal } \\
\text { versus } \\
\text { Intact, } \\
\text { external } \\
\text { control } \\
\text { P }\end{array}$} \\
\hline & & Std. dev & $\begin{array}{l}\text { Intact } \\
\text { Internal } \\
\text { control }\end{array}$ & Std. dev & & $\begin{array}{l}\text { Intact } \\
\text { External } \\
\text { control }\end{array}$ & Std. dev & & \\
\hline RF (PU) & 95.6 & 92.1 & 23.2 & 11.9 & 0.08 & 39.7 & 33.1 & 0.06 & 0.37 \\
\hline BZ (PU) & 3.4 & 0.84 & 3.8 & 1.47 & 0.89 & 7.3 & 3.46 & $0.002^{*}$ & $0.015^{*}$ \\
\hline MF (PU) & 104.7 & 93.0 & 89.2 & 64.9 & 0.89 & 66.5 & 44.1 & 0.446 & 0.49 \\
\hline$t_{\mathrm{RF}}(\mathrm{s})$ & 131.4 & 70.2 & 17.8 & 15.9 & $0.043^{*}$ & 24.0 & 19.0 & $<0.001^{*}$ & 0.54 \\
\hline$t_{\mathrm{MF}}(\mathrm{s})$ & 191.6 & 99.2 & 125.6 & 172.5 & 0.5 & 92.4 & 48.1 & $0.019^{*}$ & 0.54 \\
\hline$t_{\mathrm{HR}}(\mathrm{s})$ & 17.3 & 14.6 & 44.8 & 14.3 & 0.14 & 73.6 & 28.9 & $<0.001^{*}$ & 0.062 \\
\hline Ratio MF/RF & 1.39 & 0.5 & 4.76 & 3.25 & $0.008^{*}$ & 2.42 & 1.19 & $0.004^{*}$ & $0.049^{*}$ \\
\hline
\end{tabular}

* Denotes a statically significant difference. Abbreviations: RF = Resting flux, BZ = Biological zero, MF = Maximum flux, $t_{\mathrm{RF}}$ $=$ time to resting flux, $t_{\mathrm{MF}}=$ time to $\mathrm{MF}, \boldsymbol{t}_{\mathrm{HR}}=$ time to half recovery, $\mathrm{PU}=$ Perfusion units, SEM $=$ Standard error mean .

\section{Discussion}

This study demonstrated significant differences in microcirculation, assessed as $\mathrm{PORH}$, between ruptured healing and intact AT. The typical PORH response in the intact AT started with a fast rise in flux value, highly over resting flux, followed by a quick recovery to baseline. Comparing this response with the healing AT, the difference in character was easily detected by graphically viewing the data, where $\mathrm{PORH}$ response never reached over resting flux. Such differences in hyperemic response may suggest a susceptibility to eg. repetitive trauma.

The observed impairment of the microcirculation in the ruptured AT as compared to intact AT at two weeks post-rupture is strengthened by earlier experimental studies. Thus, a study of experimental Achilles tendon ruptures demonstrated at two weeks postinjury $38 \%$ lower resting blood flow to the gastrocnemius muscle and concluded that the circulation to the tendon is not adequately restored ${ }^{13}$.

The consistent results, observed as similar $\mathrm{PORH}$ response curves in intact versus ruptured AT, obtained by using invasive LDF together with good reproducibility results from earlier studies ${ }^{12}$ suggest that LDF is a good method for measuring the human microcirculation also in the Achilles tendon. The invasive LDF technique is a highly sensitive method, which can measure the smallest change in the blood flow. Combined with the fact that it records in a small volume, approximately $1 \mathrm{~mm}^{3}$, makes it a vigorous method to measure microcirculation. But the high-end accuracy comes with a precaution as it is vulnerable to external movement such as tension of the cable, small body movements, contraction of muscles when occluding the leg or movement of the probe. However, with a standardized protocol the invasive LDF method becomes a good and reproducible method for evaluating the $\mathrm{PORH}$ response, as demonstrated previously ${ }^{12}$. 
Earlier studies on tendon microcirculation has mainly focused on non-invasive laser Doppler measurements demonstrating that microcirculatory blood flow is significantly elevated at the point of pain in insertional and midportion tendinopathy ${ }^{18}$. Moreover, it was demonstrated that an eccentric-training program performed daily over 12 weeks reduced the increased paratendinous capillary blood flow in Achilles tendinopathy ${ }^{19}$. Another approach to measure tendon microcirculation has been attempted by real-time harmonic contrastenhanced ultrasound demonstrating that this method can detect an increased microvascular volume in healthy tendon tissue in response to a 1 hour run exercise $^{20}$. Another study using non-invasive laser Doppler showed no increase in blood flow following Achilles tendon exercise 21 . Whether invasive LDF could provide more precise measurement on local blood is not known. Earlier studies, however, using invasive intratendinous LDF demonstrated that male gender, advancing age and mechanical loading of the tendon are all associated with diminished tendon blood flow ${ }^{9}$. Moreover Åström ${ }^{9}$ demonstrated that degenerative Achilles tendinopathy is associated with hyperemia.

The difference in $\mathrm{PORH}$ between ruptured and intact AT, observed as prolonged time to resting flux and decreased ratio of maximum- and resting flux, should be attributed to the lack of a post-occlusive hyperemic peak during early healing after acute ATR. This could be due to the fact that the healing tendon is already hyperemic and that the vessels are already fully dilated in the resting state. Therefore, the capability to further react with increased blood flow following an ischemic period is reduced. This theory is supported by histological findings of increased vascularity, ede$\mathrm{ma}$ and is hyperemia in healing rabbit and rat tendons after rupture 22 . The higher baseline blood flow, i.e. resting flux, seen in the healing AT in this study, that nearly reached statistical significance, also points in this direction.

The observation of a prolonged time to resting flux in the ruptured AT may also be indicative of an impaired peripheral arterial flow in ATR patients. This suggestion is strengthened by a study demonstrating that "time to resting flux" is the best parameter to classify patients with peripheral arterial obstructive disease ${ }^{12}$. Moreover, the findings of a lower ratio of maximumand resting flux in the ruptured AT is also consistent with the results seen in patients with peripheral arterial obstructive disease ${ }^{12}$.

This study also noted differences in microcirculation of the intact tendons between the external- and the internal controls. Such disparities could partly be due to microcirculatory changes also on the contralateral side, similarly as have been noted in fracture healing $^{23}$. Another explanation may be the age difference between the groups causing degenerative microcirculatory changes, as has been indicated in earlier studies $^{9,24,25}$. Previous studies have also demonstrated a higher risk of AT-rupture on the contralateral side of a ruptured leg and biopsies confirmed degeneration on the contralateral $\mathrm{AT}^{9,25}$. It may prove that microcircu- latory LDF-data on Achilles tendons may predict degenerative changes, hypothetically the extrinsic microcirculation may also predict an increased risk for rupture. However, such conclusions warrant further studies.

A possible limitation of the study was the fact that the microcirculatory data from this study were only obtained from the midportion of the Achilles tendon and thus we cannot comment on the blood flow in either the bone tendinous- or musculo-tendinous junction. However an earlier study demonstrated a lower perfusion at the insertion but otherwise a stable distribution of blood flow along the Achilles tendon ${ }^{9}$. It should also be noted that the external control patients differed regarding age as compared to the Achilles tendon rupture patients. The ruptured patient group exhibited a mean age 36.6 years while the healthy volunteers group had a mean age of 27.1 years. The patients with AT rupture are representative of the population, which is subjected to this type of injury both in age and sex. The lower age of the healthy volunteers makes them suitable as external controls since they are less likely to exhibit tendon degeneration and they demonstrated no history of tendon pathology.

In conclusion this study demonstrates a feasible methodology to measure the in vivo vascular microcirculation in human Achilles tendon during healthy and healing conditions. A clear difference in microcirculatory response was noted between healthy and healing Achilles tendons. Moreover, we showed a difference in microcirculation between the two intact control groups, which differ in age, suggesting degenerative changes on the tendon contralateral to the ruptured side. Whether measurements of microcirculatory response in intact and healing Achilles tendons can be used to predict a vulnerability to injury and capacity to full reparative response should be assessed in future studies.

\section{Conflict of interests}

The Authors declare that they have no conflict of interests regarding the publication of this paper.

\section{References}

1. Haggmark T, Eriksson E. Hypotrophy of the soleus muscle in man after achilles tendon rupture. Discussion of findings obtained by computed tomography and morphologic studies. Am J Sports Med. 1979;7(2):121-126.

2. Nilsson-Helander K, Silbernagel KG, Thomee R, et al. Acute achilles tendon rupture: a randomized, controlled study comparing surgical and nonsurgical treatments using validated outcome measures. Am J Sports Med. 2010;38(11):21862193.

3. Wong J, Barrass V, Maffulli N. Quantitative review of operative and nonoperative management of achilles tendon ruptures. Am J Sports Med. 2002;30(4):565-575.

4. Kjaer M, Langberg $H$, Skovgaard D, et al. In vivo studies of peritendinous tissue in exercise. Scand J Med Sci Sports. 2000;10(6):326-331. 
5. Naito M, Ogata K. The blood supply of the tendon with a paratenon. An experimental study using hydrogen washout technique. The Hand. 1983;15(1):9-14.

6. Carr AJ, Norris SH. The blood supply of the calcaneal tendon. The Journal of bone and joint surgery British volume. 1989;71 (1):100-101.

7. Chen TM, Rozen WM, Pan WR, Ashton MW, Richardson MD, Taylor GI. The arterial anatomy of the Achilles tendon: anatomical study and clinical implications. Clinical anatomy. 2009;22(3):377-385.

8. Theobald P, Benjamin M, Nokes L, Pugh N. Review of the vascularisation of the human Achilles tendon. Injury. 2005;36 (11):1267-1272.

9. Astrom M. Laser Doppler flowmetry in the assessment of tendon blood flow. Scand J Med Sci Sports. 2000;10(6):365-367.

10. Yvonne-Tee GB, Rasool AH, Halim AS, Wong AR, Rahman AR. Method optimization on the use of postocclusive hyperemia model to assess microvascular function. Clinical hemorheology and microcirculation. 2008;38(2):119-133.

11. Cracowski JL, Gaillard-Bigot F, Cracowski C, Roustit M, Millet C. Skin microdialysis coupled with laser speckle contrast imaging to assess microvascular reactivity. Microvascular research. 2011;82(3):333-338.

12. Morales F, Graaff R, Smit AJ, et al. How to assess post-occlusive reactive hyperaemia by means of laser Doppler perfusion monitoring: application of a standardised protocol to patients with peripheral arterial obstructive disease. Microvascular research. 2005;69(1-2):17-23.

13. Jozsa L, Kannus $P$, Jarvinen TA, Balint J, Jarvinen M. Blood flow in rat gastrocnemius muscle and Achilles tendon after Achilles tenotomy. Eur Surg Res. 1998;30(2):125-129.

14. Domeij-Arverud E, Labruto F, Latifi A, Nilsson G, Edman G, Ackermann PW. Intermittent pneumatic compression reduces the risk of deep vein thrombosis during post-operative lower limb immobilisation: a prospective randomised trial of acute ruptures of the Achilles tendon. The bone \& joint journal. 2015;97-B(5):675-680.

15. Padulo J, Oliva F, Frizziero A, Maffulli N. Muscles, Ligaments and Tendons Journal. Basic principles and recommendations in clinical and field science research. MLTJ. 2013;3(4):250252.

16. Boesen AP, Boesen MI, Torp-Pedersen S, et al. Associations between abnormal ultrasound color Doppler measures and tendon pain symptoms in badminton players during a season: a prospective cohort study. Am J Sports Med. 2012;40(3):548555.

17. Oberg PA. Laser-Doppler flowmetry. Critical reviews in biomedical engineering. 1990;18(2):125-163.

18. Knobloch K, Kraemer R, Lichtenberg A, et al. Achilles tendon and paratendon microcirculation in midportion and insertional tendinopathy in athletes. Am J Sports Med. 2006;34(1):92-97.

19. Knobloch K, Kraemer R, Jagodzinski M, Zeichen J, Meller R, Vogt PM. Eccentric training decreases paratendon capillary blood flow and preserves paratendon oxygen saturation in chronic achilles tendinopathy. The Journal of orthopaedic and sports physical therapy. 2007;37(5):269-276.

20. Pingel J, Harrison A, Suetta C, Simonsen L, Langberg H, Bulow J. The acute effects of exercise on the microvascular volume of Achilles tendons in healthy young subjects. Clinical physiology and functional imaging. 2013;33(4):252-257.

21. Kubo K, Ikebukuro T, Maki A, Yata H, Tsunoda N. Time course of changes in the human Achilles tendon properties and metabolism during training and detraining in vivo. European journal of applied physiology. 2012;112(7):2679-2691.

22. Dahl J, Li J, Bring DKI, Renstrom P, Ackermann PW. Intermittent pneumatic compression enhances neurovascular ingrowth and tissue proliferation during connective tissue healing: a study in the rat. J Orthop Res. 2007;25(9):1185-1192.

23. Rhinelander FW, Phillips RS, Steel WM, Beer JC. Microangiography in bone healing. II. Displaced closed fractures. J Bone Joint Surg Am. 1968;50(4):643-662 passim.

24. Hudlicka O. Microcirculation in skeletal muscle. Muscles Ligaments Tendons J. 2011;1(1):3-11.

25. Cetti R, Junge J, Vyberg M. Spontaneous rupture of the Achilles tendon is preceded by widespread and bilateral tendon damage and ipsilateral inflammation: a clinical and histopathologic study of 60 patients. Acta orthopaedica Scandinavica. 2003;74(1):78-84. 